\title{
Dendritoma therapy of transferrable brain glioma in mice: An experimental study
}

\author{
Ning Jiang ${ }^{1}$, Changwu Dou*2, Fuming Tian $^{2}$, Xiangdong Zhao ${ }^{2}$ \\ ${ }^{1}$ Department of Neurosurgery, The Third Affiliated Hospital of Inner Mongolia Medical University, Baotou, Inner Mongolia, \\ China \\ ${ }^{2}$ Department of Neurosurgery, The First Affiliated Hospital of Inner Mongolia Medical University, Baotou, Inner Mongolia, \\ China
}

Received: August 12, 2015

DOI: $10.14725 /$ dcc.v2n4p6
Accepted: October 20, $2015 \quad$ Online Published: December 10, 2015

URL: http://dx.doi.org/10.14725/dcc.v2n4p6

\begin{abstract}
Glioma is the most common central nervous system primary tumor, accounting for about $40 \%$ of intracranial tumors. In recent years, studies have shown that the human brain is not immune-free zone. The low immunogenicity of the tumor itself, tumor-generated immunosuppressive factors, and the low immunity of tumor patients all contribute to the "immune escape" phenomenon of glioma cells. Dendritic cells (DCs), as the strongest antigen-presenting cells in the body, play an important role in tumor immunotherapy with T-cell recognizing tumor antigens as the core. Therefore, it is necessary to adopt a strategy of culturing and sensitizing DC cells in vitro to increase their number and improve their function before returning to the body for treatment of patients with glioma.
\end{abstract}

Key Words: Glioma, Intracranial tumors

Brain glioma is the most common primary tumor in central nervous system, accounting for $40 \%$ of all encephalic tumors. Studies in recent years indicate that the human's brain is not the immunological privileged region. The "immune escape" phenomenon of brain glioma cell is caused by the following reasons: low immunogenicity of the tumor itself, immunosuppressive factor generated by the tumor and the low immunity of patients suffered from tumor. ${ }^{[1]}$ As the strongest antigen presenting cells, dendritic cells (DCs) play an important role in the tumor immunotherapy, which take $\mathrm{T}$ cells recognizing the tumor antigen as the core. ${ }^{[2]}$ Thus, to treat the patients who suffered from the brain glioma, it is necessary to increase the DCs number and improve their function during being cultured and sensitized in vitro, and then transfuse them in vivo.

\section{Materials and methods}

\subsection{Induction of DCs}

Peripheral blood sample was collected from the patient at $40 \mathrm{ml}$ and diluted with equivalent PBS, added with lymphocyte separation medium and centrifuged at 2,000 r/min for $20 \mathrm{~min}$. After took it out slowly, the liquid in the centrifugal tube was divided into four distinct layers from top to bottom: serum layer, mononuclear cell layer (white, tinny muddy), lymphocyte separation medium layer, and red blood cell layer. The whole mononuclear cells were extracted carefully from the centrifugal tube with the $1 \mathrm{ml}$ pipettor and transferred into the cell culture bottle, added with $2 \mathrm{ml}$ diluted rhGM-CSF and IL-4 respectively. The liquid was changed for half-dose every two days, rhGM-CSF and IL-4 were also added at $1 \mathrm{ml}$ respectively.

${ }^{*}$ Correspondence: Changwu Dou; E-mail: douchangwu @ sina.com; Address: Department of Neurosurgery, The First Affiliated Hospital of Inner Mongolia Medical University, Baotou, Inner Mongolia, China 


\subsection{Preparation of Dendritoma}

U251 cells at logarithmic growth phase were freezed and thawed repetitively for five times at $-80^{\circ} \mathrm{C}$ and indoor temperature, and then were centrifuged at $8,000 \mathrm{r} / \mathrm{min}$ for 10 min. The supernatant was collected and sterilized by filtration. DCs cultured for 10 days were collected and added with frozenthawed antigen to the proportion of DCs and C6 tumor antigen at the rate of 1:20 (the amount of antigen was counted as the tumor cells before freeze-thaw, the DCs were counted as the cells). The final concentration of DCs was $5 \times 10^{5} / \mathrm{ml}$, cells were cultured in $\mathrm{CO}_{2}$ constant temperature incubator with $5 \% \mathrm{CO}_{2}$ at $37^{\circ} \mathrm{C}$ for $48 \mathrm{~h}$.

\subsection{Establishment of nude mice glioma model}

Thirty-two female BALB/c nude mice were divided into two groups, weighting 18.3-23.9 g, aged 5 weeks. Operations were processed in laminar superclean bench. The U251 cells were dissociated and the concentration was adjusted. The scalp of the mice was cut through the intersection between the inner canthus and sagittal midline of the head at $0.5 \mathrm{~cm}$ lengthways. The two side cuticles were separated, and the skull was exposed and drilled. Cell suspension containing $2 \times 10^{6}$ C6 was injected at $20 \mu \mathrm{l}$ with $20 \mu \mathrm{l}$ microinjector, inserting vertically, $5 \mathrm{~mm}$ depth (to endocranium). injection time was approximately $10 \mathrm{~s}$ (avoid injection too fast). The needle was pulled slowly, the hole was sealed with sterile bone wax and sterilized with iodine volts alcohol after suture.

\subsection{Test grouping and intervention}

Thirty-two mice were divided into two groups randomly (A: control group with saline solution; B: treatment group with DCs), with 16 mice in each group. Each group was divided into another two groups (1 and 2) randomly, with 8 mice in each group. Group 1: measuring the area ratio of the tumor necrosis to the tumor from the maximum section; Group 2: recording the survival time of the mice. On the $11^{\text {th }}$ day after bearing tumor in mice, the treatment group was injected with sensitized DCs by abdominal subcutaneous injection, with the amount of $4.6 \times 10^{5}$. The injection was done once again one week later; the treatment group was injected with equivalent saline solution in the meantime. The growth conditions and the clinical symptoms of the mice were observed and recorded. On the $18^{\text {th }}$ day, mice in group 1 were anesthetized with diethyl ether and the left ventricle was pierced after thoracotomy, $10 \%$ paraformaldehyde was poured for two hours, then the brain tissue was isolated for pathological slices preparation and HE staining. The natural survival time of all mice in group 2 was also recorded.

Published by New Century Science Press

\subsection{Pathological slices preparation and HE staining}

The brain tissue was put into $10 \%$ formaldehyde solution and fixed for 24 hours. The coronal slice was made through the maximum section of the tumor. Dehydrated, waxed, embedded and sliced with the thickness of $5 \mu \mathrm{m}$ through the maximum coronal plane. Each slice was dewaxed and stained with hematoxylin and eosin, sealed with gum and observed by microscope. Image analysis was done with the Olympus CX41 computer system. Delineated the area and calculated with specific software analysis system, then recorded the data and calculated the ratio.

\section{Results}

\subsection{Morphological observation of DCs}

Cytokines were added into the culture bottle every three days. Some small processes appeared on cells surface from the $4^{t h}$ day. On the $8^{t h}-10^{t h}$ day, the processes increased and became dendritic on some cells surface. The cells dispersed from the state of aggregation step by step and shed from the bottle wall. The cells appeared classic dendritic on the $12^{\text {th }}$ day. The phenotype of DCs was proved mature by flow cytometer.

\subsection{Symptom observation of the tumor-bearing mice}

An average of around 20 days, the obvious clinical symptoms appeared after tumor-bearing in mice, which included: activity reduced obviously, slow response to the sound and photic stimulation, bulging eyes, increasing skin fold, old appearance, unstable body when crawling, reduced eating and drinking.

\subsection{General situation and pathological slice exami- nation}

The mice were anesthetized and fixed. Thoracotomy was performed to isolate the brain tissue carefully. The residual trace and ridgy tumor could be seen from the surface of the tissue (see Figure 1A). The brain midline appeared skew in the coronal section. The quasi-circular tumor tissue with vessels could not be distinguished from the normal brain tissue and extended around (see Figure 1B). Microscope observation: the tumor cells with different sizes and shapes could be distinguished from the normal cells easily through the high power lens (see Figure 2A); the vessels in the tumor tissue and the necrotic zone in the middle could be seen through the low power lens (see Figure 2B). 


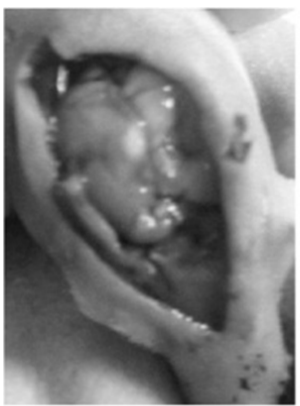

A

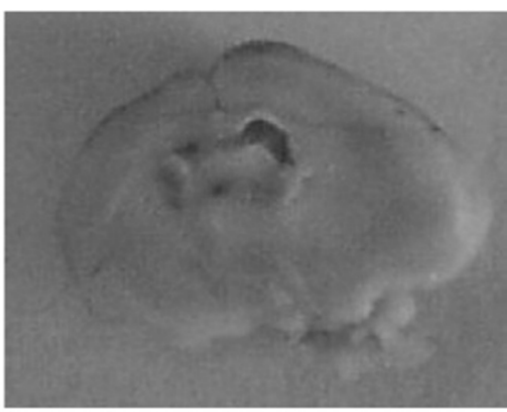

B
Figure 1: The U251 cells injection into the mice's brain on the $20^{\text {th }}$ day

\subsection{The areas of tumor and necrosis in treatment and control groups}

$T$-test analysis indicated that statistical significance between the treatment and control groups was established at $p<.05$. The area ratio of the necrosis to the tumor in treatment group was bigger than the one in control group (see Table 1).

\subsection{The contrast of the survival time in mice}

Treatment group with abdominal hypodermic injection: the survival time for the 8 mice was $33 \mathrm{~d}, 46 \mathrm{~d}, 39 \mathrm{~d}, 49 \mathrm{~d}, 50$ d, 55 d, 43 d, 34 d respectively; Control group with normal saline: the survival time was $16 \mathrm{~d}, 20 \mathrm{~d}, 17 \mathrm{~d}, 30 \mathrm{~d}, 26 \mathrm{~d}$, $32 \mathrm{~d}, 38 \mathrm{~d}$ and $28 \mathrm{~d}$, respectively. Statistical significance was established at $p<.05$. The survival time of mice in treatment group was longer than the ones in control group.
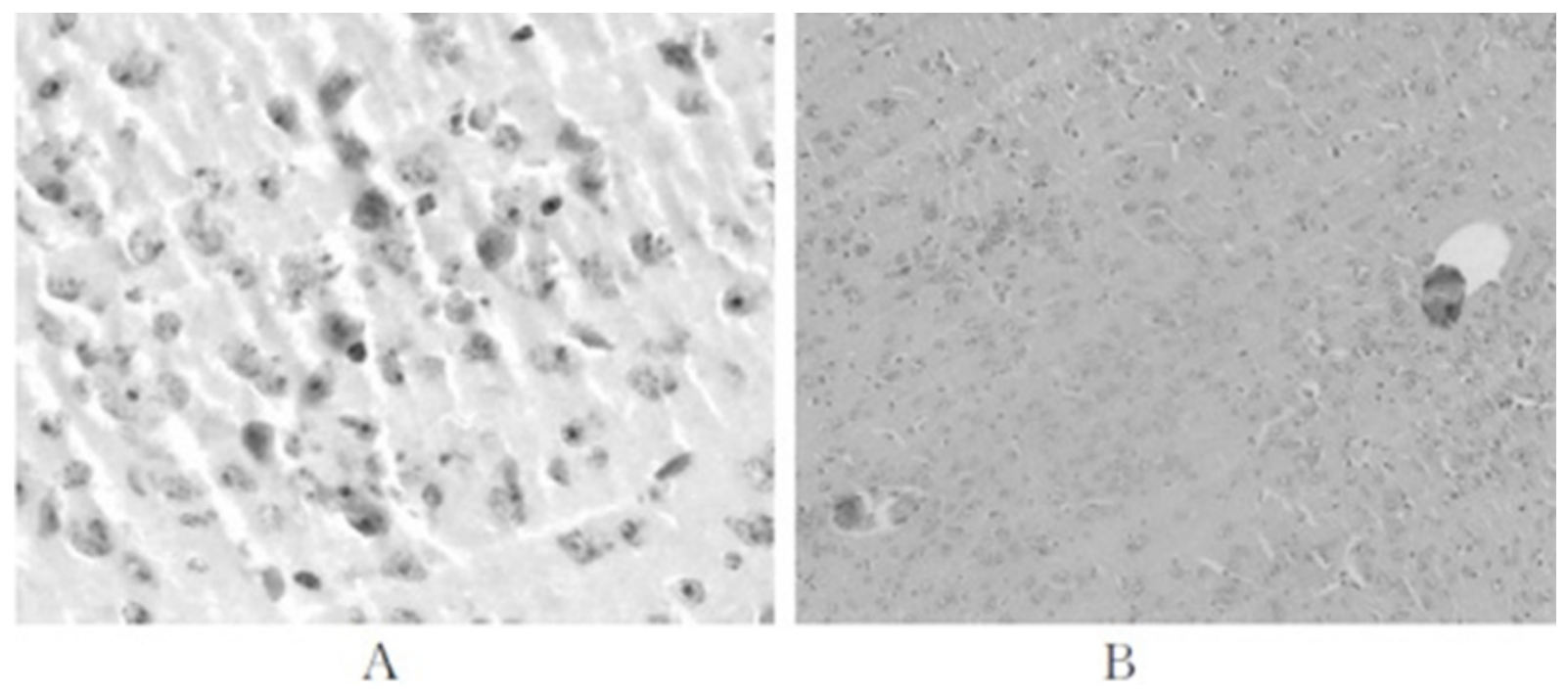

\section{Discussion}

As the strongest antigen presenting cell known so far, DCs could ingest and process the antigen, express high-level MHC molecules, costimulatory molecules and adhesion molecules. $^{[3]}$ In our study, a certain number of DCs was cultured in vitro, and the mature ones sensitized by tumor antigen were injected into the tumor-bearing mice. The test simulated the detailed process of DCs generation, maturity, sensitization and killing in vivo.

Lots of literatures indicated three ways to induce DCs in vitro: bone marrow, peripheral blood and umbilical cord blood. It is difficult to obtain DCs from the bone marrow and umbilical cord blood, furthermore, the cells will be diverse after amplification. ${ }^{[4,5]}$ Nevertheless, the DCs from peripheral blood have many advantages, such as easy to obtain, simple and convenient, wide applicability and easy to control.

The base of this study is the establishment of the stable animal model of brain glioma, which is connected with the type of the animal, the category of the tumor, the method and location of inoculation and the growth time. ${ }^{[6]}$ The ideal animal model of brain glioma should own the following conditions: the tumor cells should be able to grow in vitro with the characteristics of the glioma and malignancy cells; the tumor's growth rate can be measured with short growth time and the lifetime is stable; the tumor model should own the similar growth characteristics of human brain glioma, such as infiltrative growth, abundant tumor vessels, and so on. The BALB/c nude mice were chosen due to the immunodeficiency, that is, the tumorigenesis would be faster after inoculating in brain and the survival rate was higher. But there is another problem, the mice have poor disease resistance and need higher feeding conditions. Thus, it is crucial to ensure the clean environment for the mice during the test and checking process.

Figure 2: The tumor's pathological characteristics 
Table 1: The data of the areas of the necrosis and tumor and their ratios in the treatment and control groups

\begin{tabular}{|c|c|c|c|c|c|c|}
\hline \multirow{2}{*}{ Item } & \multicolumn{3}{|c|}{ Treatment group } & \multicolumn{3}{|c|}{ Control group } \\
\hline & Necrosis area $\left(\mathrm{mm}^{2}\right)$ & Tumor area $\left(\mathrm{mm}^{2}\right)$ & Ratio & Necrosis area $\left(\mathrm{mm}^{2}\right)$ & Tumor area $\left(\mathrm{mm}^{2}\right)$ & Ratio \\
\hline 1 & 1.07231 & 2.78651 & 0.3848 & 1.81733 & 11.42176 & 0.1550 \\
\hline 2 & 2.80451 & 8.02147 & 0.2909 & 2.09277 & 11.71530 & 0.1786 \\
\hline 3 & 2.57331 & 7.00481 & 0.3495 & 2.13807 & 11.76112 & 0.1818 \\
\hline 4 & 2.29201 & 11.05651 & 0.2721 & 2.34552 & 11.76230 & 0.1994 \\
\hline 5 & 2.40430 & 8.83601 & 0.3674 & 0.74623 & 2.99591 & 0.2731 \\
\hline 6 & 3.47559 & 11.94597 & 0.1851 & 2.35242 & 8.61377 & 0.2217 \\
\hline 7 & 1.36298 & 7.36187 & 0.2073 & 0.60693 & 2.74976 & 0.2491 \\
\hline 8 & 2.32631 & 7.27347 & 0.3198 & 1.43623 & 7.23303 & 0.1986 \\
\hline
\end{tabular}

Note. $T$-test was used for two independent samples, $p<.05$ showed significant differences in the area ratio

After the establishment of glioma model, the choice of treatment beginning is especially important, that is, before the exponential growth phase of the tumor, and the treatment outcome could be better determined with the intervention during this period. The study indicated that the tumorigenesis happened on the $10^{\text {th }}$ day after the U251 cells were inoculated, and the tumor went into the exponential growth phase on the $15^{\text {th }}$ day, thus, the treatment time was the $7^{\text {th }}$ to $10^{t h}$ day after being inoculated. To ensure the rational-

\section{References}

[1] Wang DH, Li XG, Li G, et al. The experimental study on anti-glioma with the dendritic cell fused cell vaccine. Chinese Journal of Neurosurgery. 2006; 22(11): 699-701.

[2] Fu Y, Zhao G, Zhang WL, et al. The preparation and antineoplastic activity of the fusion cell with DC and glioma. Chinese Journal of Laboratory Diagnosis. 2006; 5(10): 468-470.

[3] Meng FD, Wang XH, Wang Y, et al. The study of the interaction between the dendritic cell cultured in vitro and killer cells induced by ity of the time, the treatment was started from the $7^{\text {th }}$ day in the study. With the precise experimental ideas and processes, the study plays an important role in the preparation of DCs cellular vaccine and the transition from tumor gene therapy to clinical application in the future.

\section{Conflicts of Interest Disclosure}

The authors have no conflicts of interest related to this article.

cytokines. Chinese Journal of Gerontology. 2006; 6(26): 818-820.

[4] Santegoets SJ, van AJ, van AA. Human dendritic cell line models for DC differentiation and clinical DC vaccination studies. Journal of Leukocyte Biology. 2008; 84(7): 1-10. https ://doi .org/10. $1189 / j 1 b .0208092$

[5] Ishimoto H, Yanagihara KS. Single-cell Observation of Phagocytosis by human Blood Dendritic cells. JPN J Infect Dis. 2008; 61(7): 294-297. PMid: 18653972.

[6] Meng QH, Bo YL, Gong AJ, et al. The brain glioma therapy with dendritic cell vaccines in rat: An experimental study. Chinese Journal of Neurosurgery. 2006; 22(11): 055-059. 